\title{
Influence of dc bias voltage on the refractive index and stress of carbon-diamond films deposited from a $\mathrm{CH}_{4} / \mathrm{Ar}$ rf plasma
}

\author{
Gehan A. J. Amaratunga and S. Ravi P. Silva \\ Engineering Department, Cambridge University, Trumpington Street, Cambridge CB2 1PZ, \\ United Kingdom \\ David R. McKenzie \\ Department of Applied Physics, University of Sydney, NSW 2006, Australia
}

(Received 22 April 1991; accepted for publication 9 August 1991)

\begin{abstract}
The dc self bias voltage developed during $\mathrm{CH}_{4} / \mathrm{Ar}$ radio frequency plasma-enhanced vapor deposition of thin films containing polycrystalline diamond grains within an $a: \mathrm{C}$ matrix (carbon-diamond) is found to influence the optical and mechanical properties of the films. In particular it is shown that there is a simultaneous etch deposition process which takes place, and that the dc bias can be used to control this etch rate, and hence the net film growth rate. When a balance between etching and deposition is achieved, the films show increased residual stress and optical density with exposure to $\mathrm{Ar}^{+}$bombardment in the plasma. In addition to the measured dc bias the local electric field developed around the substrate is also found to significantly influence the energy with which ions impinge upon the growing film.
\end{abstract}

\section{INTRODUCTION}

The properties of wide band gap, low dielectric constant, high intrinsic electrical resistivity, high-carrier mobility, high thermal conduction, and chemical inertness make diamond an attractive material for electronic devices which are to operate in hostile environments. ${ }^{1}$ In addition its extreme hardness and optical transparency make it attractive in a host of coating applications. ${ }^{2,3}$ The recently discovered possibility of depositing polycrystalline diamond films on different substrate materials has made the prospect of realizing the technological potential of diamond more realistic.

Diamond films can be deposited using a variety of plasma-enhanced chemical vapor deposition (PECVD) techniques. These include plasmas formed by coupling $\mathrm{dc}$, rf, or microwave electric fields to a gas mixture which contains $\mathrm{CH}_{4}$ with either $\mathrm{H}_{2}$ or Ar. In this paper we concentrate on films formed from a $\mathrm{CH}_{4} / \mathrm{Ar}$ rf plasma. The rf PECVD technique has the advantages of being a low-temperature process where substrates are commonly held at $20^{\circ} \mathrm{C}$ and easily extendable for large area deposition. The major disadvantage is that the films obtained from a $\mathrm{CH}_{4} / \mathrm{Ar}$ rf plasma are not comprised entirely of diamond grains, but rather diamond grains within an $a: \mathrm{C}$ matrix and is therefore a mixed phase material. ${ }^{4}$ This material is therefore more accurately described as carbon-diamond as opposed to diamond or diamond-like carbon. Transmission electron microscope (TEM) shows the diamond grains in carbon-diamond films to range from 10 to $200 \mathrm{~nm}^{4}$ Surface microanalysis carried out using scanning tunnelling microscopy (STM) also confirm the diamond- $a: C$ mixed phase nature of the films. ${ }^{5}$ Scanning electron microscope (SEM) examination of carbon-diamond film surface shows it to be very smooth and free of pin holes or surface cracks. Surface roughness measurements using a profilometer reveal that features of $5-10 \mathrm{~nm}$ over scan lengths of several centimeters can be obtained for some films. This mirror quality of surface smoothness, which can be obtained in rf-deposited carbon-diamond films, is directly related to the small diamond grain size in the films. The more pure diamond films obtained from microwave PECVD typically have grain sizes of 1 to $10 \mu \mathrm{m}$ and do not result in such smooth surfaces. It is interesting to note that attempts to reduce the grain size and improve surface smoothness in microwave PECVD diamond films for x-ray lithography also led to a degradation in the purity of the films. ${ }^{6}$

In this work emphasis is placed on how the mechanical and optical properties of rf-deposited carbon-diamond films change as a function of the plasma-substrate dc self bias voltage and substrate surface area. In particular the variation of refractive index and stress in the films is characterized. The optical absorption characteristics of the films are also discussed briefly.

\section{EXPERIMENT}

The carbon-diamond films were deposited in a capacitatively coupled $13.56-\mathrm{MHz}$ rf deposition chamber at a pressure of $300 \mathrm{mT}$. A gas flow of $160 \mathrm{sccm}$ was used with an $8 \% \mathrm{CH}_{4}$ mixture in Ar. Substrate material was $\{100\}$, 1-5 $\Omega \mathrm{cm} n$-type $\mathrm{Si}$. The dc self bias which accelerates the ions taking part in film growth was controlled by varying if power input to the chamber. In this system the sample is placed on the bottom electrode which is electrically driven and cooled to $20^{\circ} \mathrm{C}$. The dc bias voltage, deposition time and substrate surface area were used as variable parameters.

All substrates were subjected to an in situ preclean in an Ar plasma with dc self bias of $-450 \mathrm{~V}$. It was found that this precleaning procedure had to be carefully determined as it has the potential to cause significant damage at the Si surface, and hence the carbon-diamond/Si interface. Figure 1 shows how the preclean procedure affects the 


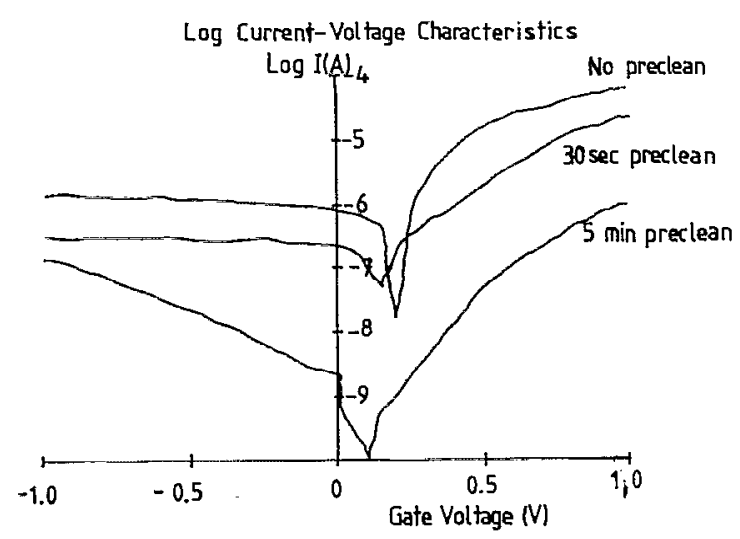

FIG. 1. Effect of Ar ion pre-cleaning Si substrates on $I-V$ characteristics of Au/Si Schottky diodes.

rectifying characteristics of Schottky diodes formed on the $\mathrm{Si}$ substrates with $\mathrm{Au}$ evaporation. Compared to the case with no plasma preclean (but with degreasing), both diodes on precleaned substrates show signs of additional defects being introduced at the $\mathrm{Au} / \mathrm{Si}$ interface. However, the sample precleaned for $30 \mathrm{~s}$ still preserves rectifying characteristics but with an increase in the junction resistance (a large ideality factor). The sample subjected to a 5-min preclean shows a loss in rectifying characteristics and an increase in the forward resistance, consistent with the presence of an excess number of defects at the $\mathrm{Au} / \mathrm{Si}$ interface.

Film thickness was determined using a Gaertner ellipsometer. The film thicknesses predicted by ellipsometry were calibrated against measurements from a Dektak 3030 profilometer. Refractive indices (ri) of the films were also obtained by ellipsometry. Film stress was obtained by mcasuring substrate curvature before and after deposition. Carbon-diamond films of up to $600 \AA$ in thickness were transparent, those in the thickness range 700-1200 $\AA$ yellow and those thicker than $1200 \AA$ a mixed blue-green and violet in color.

Figure 2(a) shows a typical infrared (IR) transmission spectrum for the carbon-diamond films, and is a useful way of characterizing the amorphous carbon present in the films. The films are transparent over a major part of the IR spectrum showing the most absorption below $1100 \mathrm{~cm}^{-1}$ $(>9 \mu \mathrm{m})$ in the far infrared. The gradual slope in the characteristic with reducing wave number down to 1200 $\mathrm{cm}^{-1}$ is introduced in the process of subtracting the $\mathrm{Si}$ substrate background from the composite IR spectrum. This type of IR spectrum is different from those reported for $a-\mathrm{C}, a-\mathrm{C}: \mathrm{H}$, and diamond-like carbon ${ }^{7-9}$ in that it shows significant absorption in the $3400-3100 \mathrm{~cm}^{-1}$ region, rather than $3100-2800 \mathrm{~cm}^{-1}$ normally associated with monohydride $\mathbf{C}-\mathrm{H}$ bonds. In the literature concerning IR studies of carbon films there is no report of the very distinct absorption peak (transmission trough) at $3225 \mathrm{~cm}^{-1}$ observed in these films. This peak could be due to the $\mathbf{H}$ bond in a triple bonded $\mathrm{C}$ chain. ${ }^{10}$ If this is the case then it is very interesting, as it shows the presence of triple bonded $C$ (carbynes) which can be considered an intermediate phase between graphite and diamond. ${ }^{11}$ Figure 2 (b) shows

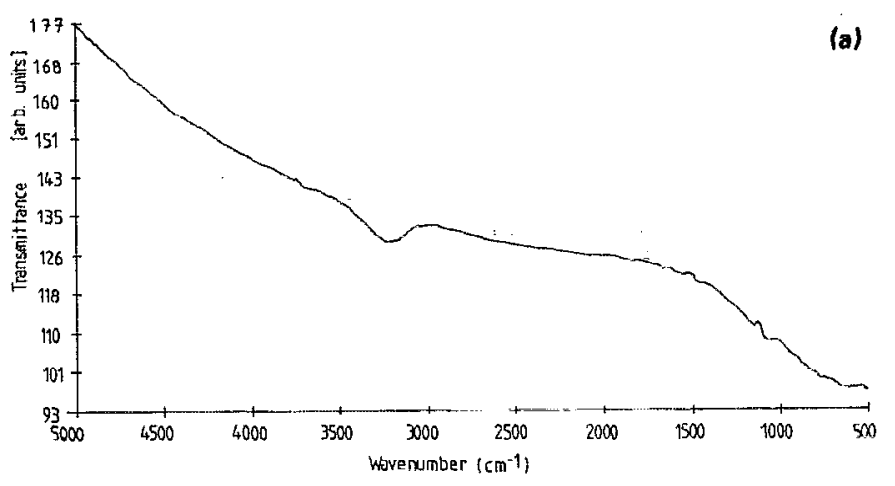

(b)

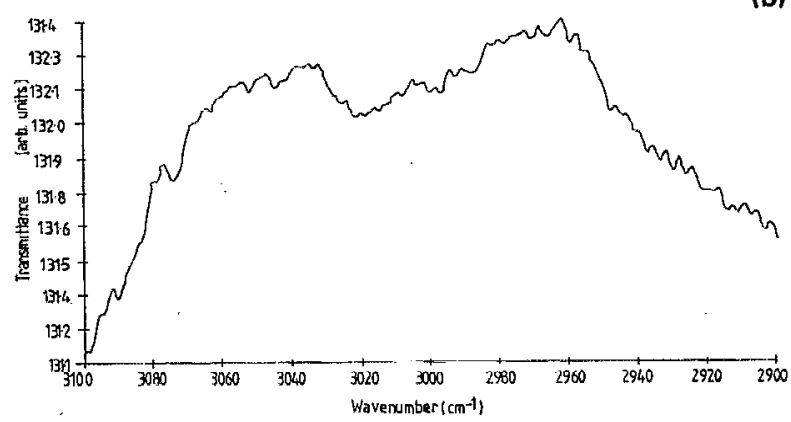

FIG. 2. (a) Typical IR transmission spectrum for a carbon-diamond film on Si with the spectrum for Si subtracted using a calibration sample: (b) Expanded IR transmission spectrum in the region normally associated with $\mathrm{C}-\mathrm{H}$ absorption.

the IR response in the region $3100-2900 \mathrm{~cm}^{-1}$ useful in determining other forms of $\mathrm{C}$ bonded to $\mathrm{H}$. Though the response in this region is much weaker, there is evidence of absorption at $3020 \mathrm{~cm}^{-1}$ and $2980 \mathrm{~cm}^{-1}$ due to aliphatic $\mathrm{C}-\mathrm{H}$ stretch, characteristic of the $\mathrm{CH}_{3}$ group. There is also some very weak absorption at $1570 \mathrm{~cm}^{-1}$ and $1430 \mathrm{~cm}^{-1}$ showing the presence of some $s p^{2}$ aromatic $\mathrm{C}-\mathrm{C}$ bonds. There is no discernible absorption at $1330 \mathrm{~cm}^{-1}$ corresponding to the $s p^{3} \mathrm{C}-\mathrm{C}$ bond. This absorption peak (transmission trough) is not though clearly seen in the IR spectra of polycrystalline diamond films or crystalline diamond. ${ }^{12}$ Pronounced IR absorption is seen below 1100 $\mathrm{cm}^{-1}$. This region is associated with aromatic $\mathbf{C}-\mathrm{H}$ bonds such as those in benzene.

An optical-UV transmission spectrum measured for a thin carbon-diamond film on $\mathrm{Si}$ using a reflection attachment on a Perkin-Elmer Lambda 17 spectrophotometer is shown in Fig. 3. The spectrum has been calibrated against the reflection spectrum from a polished $\{100\}$ Si substrate obtained from a second light beam. Two clear absorption edges at $231 \mathrm{~nm}(5.4 \mathrm{eV})$ and $300 \mathrm{~nm}(4.1 \mathrm{eV})$ are seen. The 5.4-eV absorption edge would conform to the expected optical gap due to the presence of polycrystalline diamond grains in the film, while the 4.1-eV edge could be ascribed to transitions to defect related states below the conduction band. The limited absorption above $4.1 \mathrm{eV}$ and below 5.4 $\mathrm{eV}$ suggests that the density of states between these two energies are limited. In other samples which were studied with STM and electrical band gap of $4.0-4.2 \mathrm{eV}$ was measured without any evidence of limitation in the density of 


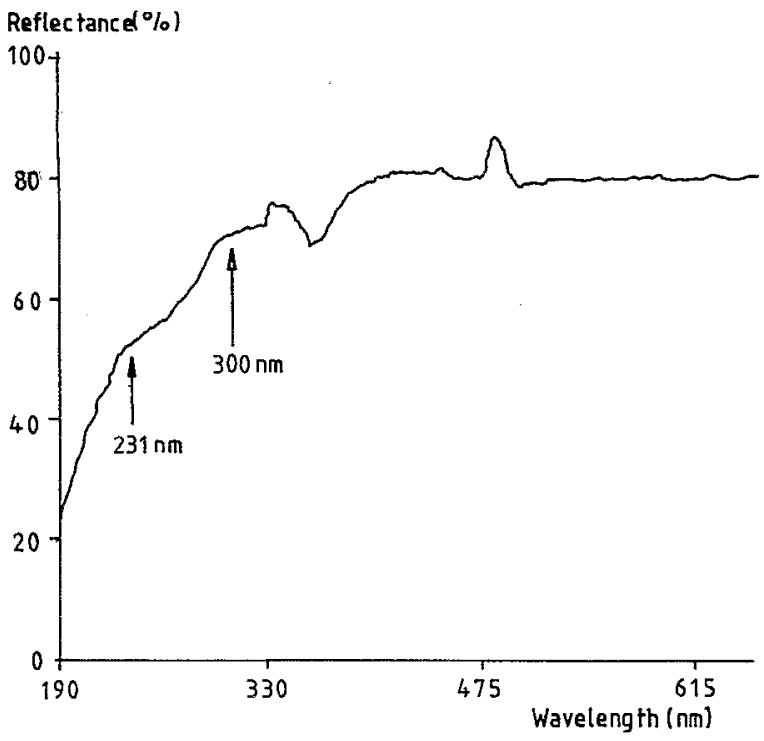

FIG. 3. Visible-UV transmission spectrum for a carbon-diamond film.

states ${ }^{5}$ in crystalline areas, indicating that it is also possible to view the 4.1-eV edge as corresponding to the band gap in the presence of significant band tailing. STM measurements also revealed the intergrain $a: C$ in carbon-diamond as having a very low band gap. It is perhaps worth noting that the single absorption edge at $4.0 \mathrm{eV}$ and the dual absorption edges at 4.0 and $5.4 \mathrm{eV}$ are seen in type-I diamonds containing nitrogen. ${ }^{3}$ Figure 4 shows a Raman spectrum of an rf-deposited $0.3-0.4-\mu \mathrm{m}$-thick carbon-diamond film, showing no discernible peak at $1332 \mathrm{~cm}^{-1}$ corresponding the shift associated with diamond. There is however a broad peak at $1580 \mathrm{~cm}^{-1}$ corresponding to $a: C$. As has been pointed out by others ${ }^{13,14}$ the absence of a Raman $1332 \mathrm{~cm}^{-1}$ response does not necessarily indicate the absence of diamond, but rather that the amorphous and $s p^{2}$ carbon content of the films is larger than 3-4\% due to the Raman scattering efficiency of graphitic carbon be-

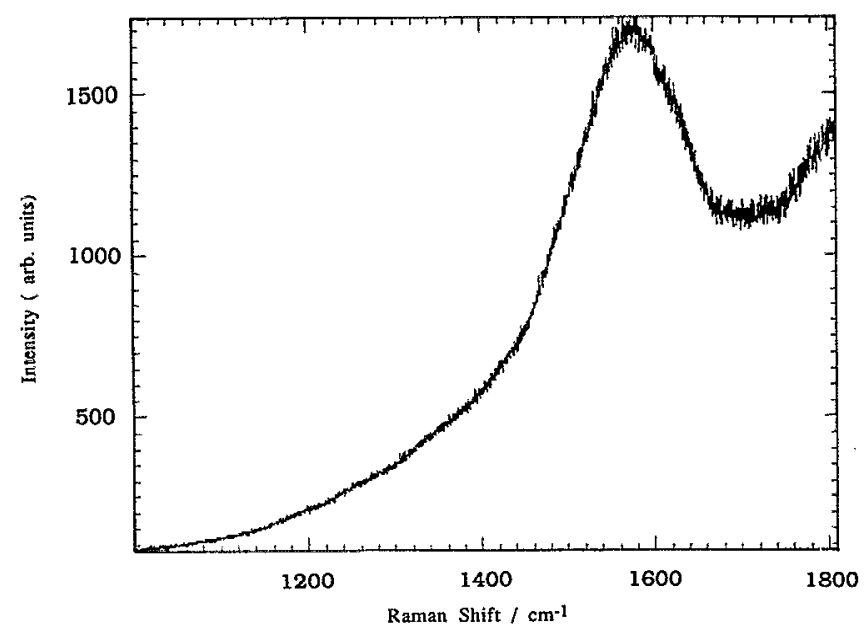

FIG. 4. Raman spectrum for a carbon-diamond film.

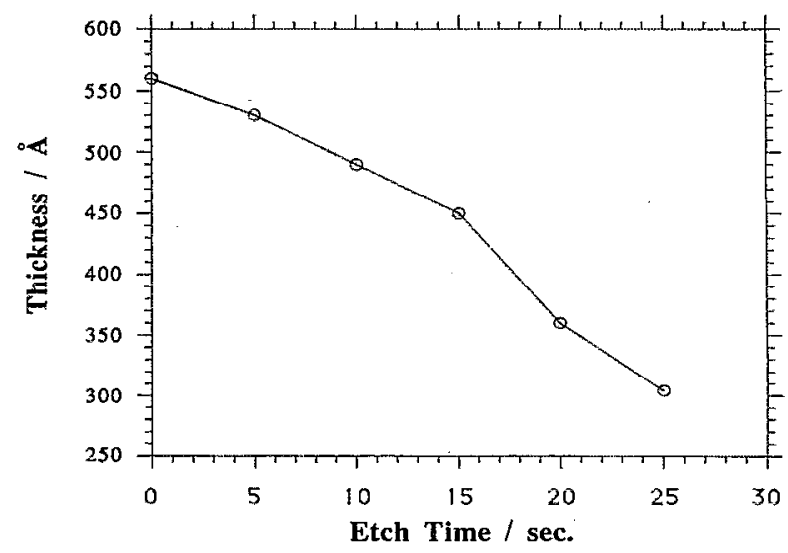

FIG. 5. Film thickness vs deposition time for different dc bias deposition conditions.

ing 30-50 times greater than that of diamond. As pointed out by Knight and White ${ }^{14}$ Raman spectroscopy is not a sensitive test for diamond in the presence of other forms of carbon. The Raman spectrum of Fig. 4 is very similar to that obtained for small grain diamond films obtained from microwave PECVD. ${ }^{6}$

\section{RESULTS AND DISCUSSION}

\section{A. Growth rate}

Figure $\mathbf{5}$ shows film thickness versus deposition time as a function of dc bias voltage. The substrate surface dimensions were $30 \mathrm{~mm} \times 20 \mathrm{~mm}$. This data gives some insight into the rf PECVD process for carbon-diamond. For the films deposited at a dc bias of $-450 \mathrm{~V}$ it is seen that after an initial step deposition of $100 \AA$ in $30 \mathrm{~s}$, the net deposition for the next $45 \mathrm{~min}$ is $70 \AA$. By contrast the films deposited at $-160 \mathrm{~V} \mathrm{dc}$ bias grow at a constant rate of 70 $\AA / \mathrm{min}$ over the entire $45 \mathrm{~min}$. The films deposited at dc biases of $-320 \mathrm{~V}$ and $-400 \mathrm{~V}$ show continued film growth, but with changes in growth rate for the $-400 \mathrm{~V}$

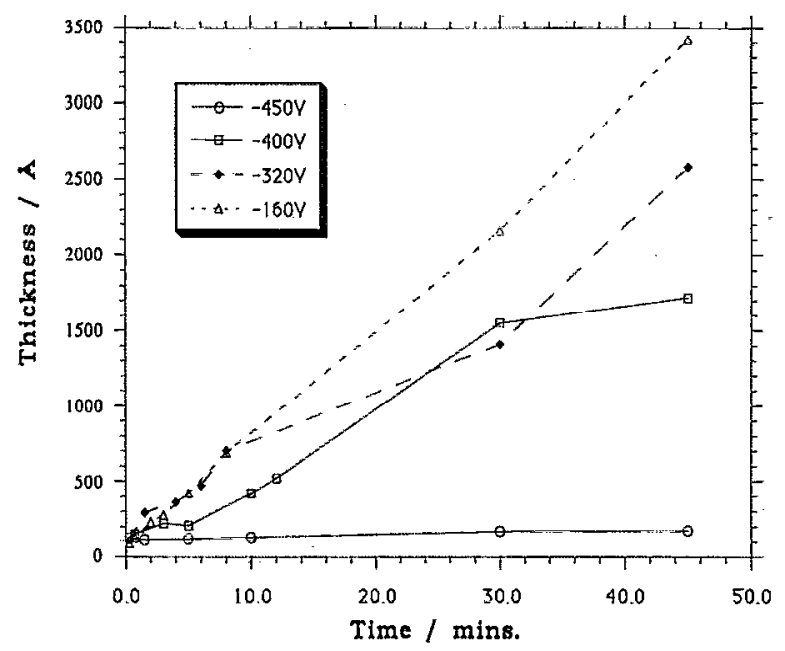

FIG. 6. Film thickness vs etch time for a film exposed to an Ar plasma. 
case as the deposition proceeds. This leads us to view $\mathrm{CH}_{4} / \mathrm{Ar}$ rf PECVD as an etch/deposition process, and that a balance between the two competing processes components can be achieved by changing the dc bias voltage. This was further confirmed by exposing carbon-diamond films to a pure $\mathrm{Ar}^{+}$plasma and measuring film thickness with time. Figure 6 shows thickness vs time for a carbondiamond film which was initially $560 \AA$ thick (deposited at a dc bias of $-400 \mathrm{~V}$ ) exposed to an $\mathrm{Ar}^{+}$plasma at -400 $\mathrm{V} \mathrm{dc}$ bias (flow rate $20 \mathrm{sccm}$, pressure $30 \mathrm{mT}$ ). The much faster etch rate seen in Fig. 6, $10 \AA / \mathrm{s}$, compared to the corresponding film deposition rate of approximately $50 \AA$ / min in Fig. 5, indicates that Ar plays a significant role in etching the growing carbon-diamond films.

It should be noted that while $\mathrm{Ar}^{+}$ions etch the growing films, the $\mathrm{dc}$ bias which influences $\mathrm{Ar}^{+}$acceleration across the sheath space, and hence the sputter efficiency, is proportional to the if power coupled into the chamber at a given pressure. Therefore a higher proportion of Ar atoms in the chamber will also be ionized with increasing $\mathrm{rf}$ power and dc bias voltage. Changes in both the dc bias voltage and ionized Ar concentration will therefore influence the etch rate of the carbon-diamond film during deposition.

\section{B. Refractive index and stress}

If we now consider the film which was deposited at a $\mathrm{dc}$ bias $-450 \mathrm{~V}$ in Fig. 5 and note that virtually no growth takes place after $15 \mathrm{~min}$ : It is reasonable to conclude that an etch/deposition balance has been achieved. During this "no growth" period the film can be viewed as being subjected to $\mathrm{Ar}^{+}$bombardment. Figure 7(a) shows the variation of refractive index (ri) with time as a function of dc bias voltage for the same films as those in Fig. 5. Figure 7 (b) shows the variation of ri with thickness for the same films. For the film deposited at $-450 \mathrm{~V} \mathrm{dc}$ bias it is seen that the ri increases with time during the no growth period. This increase in the effective optical density of the film is consistent with the compacting of the film and a rise in film stress due to $\mathrm{Ar}^{+}$bombardment. Figure 8 shows measurements of carbon-diamond films stress as a function of ri which are consistent with the view that $\mathrm{Ar}^{+}$bombardment leads to increased film stress during the no growth period. It is interesting to note that film stress values above $2 \mathrm{GPa}$ correspond to hydrostatic pressures at which diamond is the stable phase of carbon at room temperature. The significance of such high film stresses in determining the transition from graphitic amorphous carbon to amorphous diamond in ion beam deposited films has been experimentally verified recently. ${ }^{15,16}$

From Fig. 5 it can be seen that the film deposited at a de bias of $-400 \mathrm{~V}$ shows a growth rate of approximatcly $50 \AA / \mathrm{min}$ up to $30 \mathrm{~min}$, after which it drops to $20 \AA / \mathrm{min}$. From Fig. 7(a), which shows the corresponding ri data, it is seen that after an initial rapid rise to a value of 2.1 , it stays at this value up until 30-min deposition time. After this an increase in the ri which is accompanied by a fall in the growth is seen. This suggests that during the slow
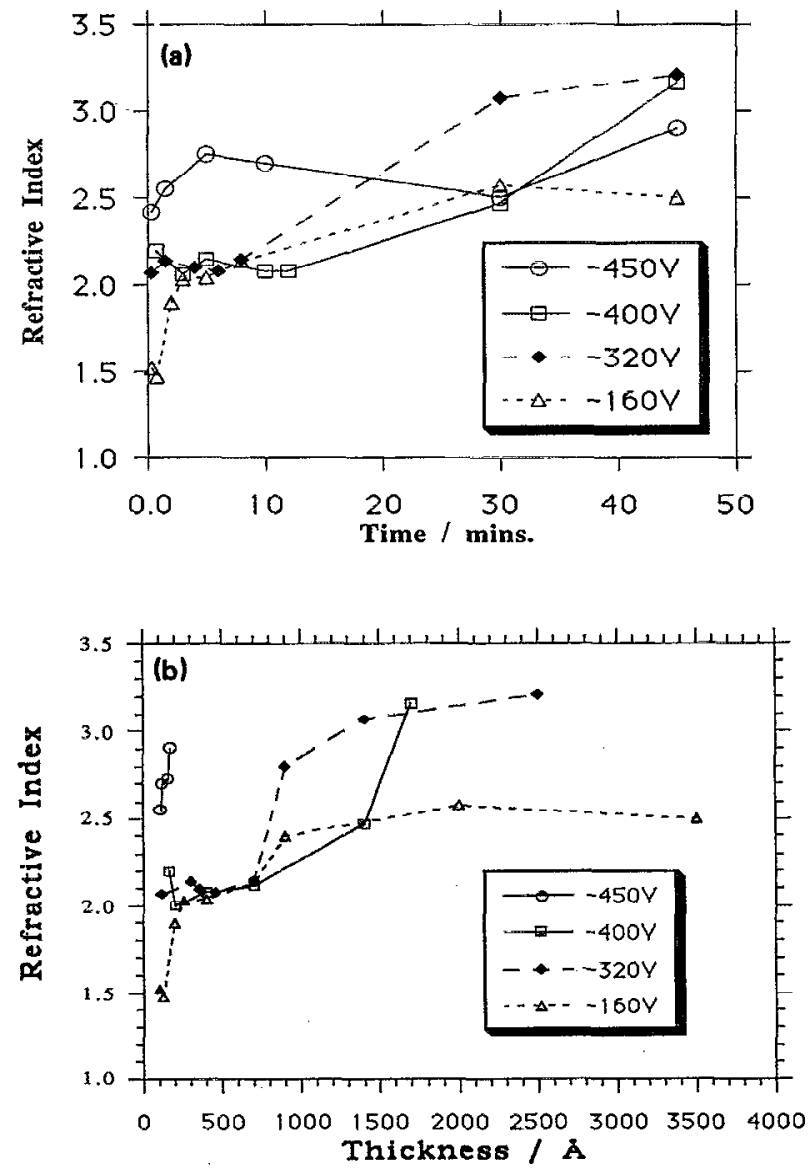

FIG. 7. (a) Refractive index vs time for different de bias deposition conditions. (b) Refractive index vs thickness for different dc bias deposition conditions.

growth rate phase this film is also being subjected to significant compaction by $\mathrm{Ar}^{+}$bombardment.

The results indicate that Ar plays a significant role in the etching cycle of this combined etch/deposition process. When the dc self bias between the rf plasma and the substrate is increased the energy with the heavy $\mathrm{Ar}^{+}$impinge on the substrate also increase. This in turn leads to an increase in the sputter yield and faster etching of carbondiamond films. The results also show that there is a tran-

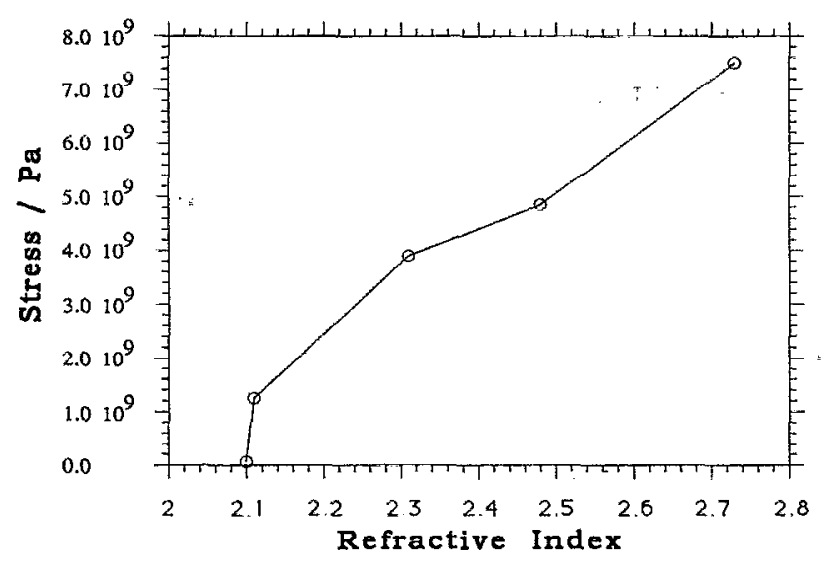

FIG. 8. Film stress vs the refractive index. 


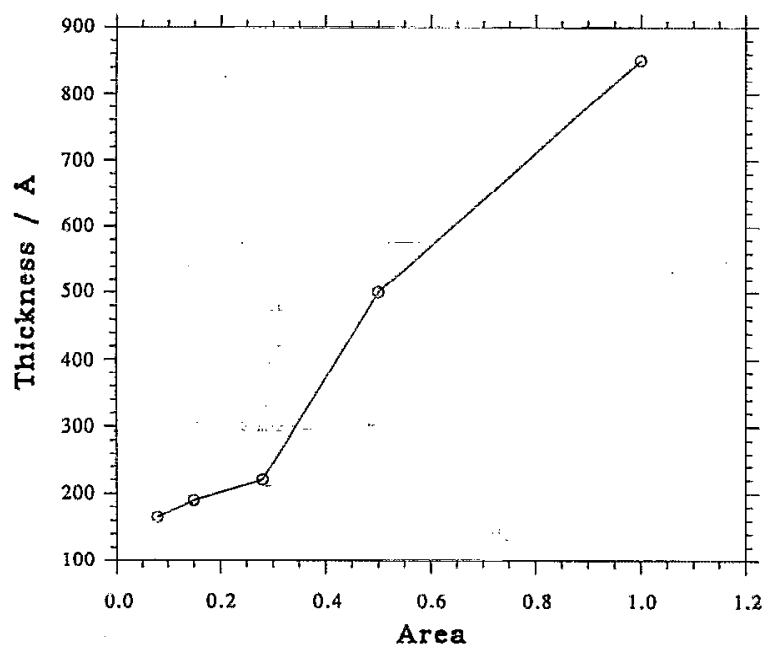

FIG. 9. Thickness vs substrate deposition area.

sition to a balance between etching and deposition at a particular film thickness, and that the thickness at which this transition occurs is a function of the dc bias. After this balance has been achieved the films are subjected to compaction or densification by $\mathrm{Ar}^{+}$bombardment which leads to an increase in film stress and ri. The absence of growth rate fall off in the films deposited at -320 and $-160 \mathrm{~V}$ dc bias show that the etch/deposition balance thickness has not been reached after $45 \mathrm{~min}$ of deposition. The ri data shows that for the $-160 \mathrm{~V}$ dc bias case there is a rapid rise to a value of 2.1 , after which it increases more slowly to a value of $2.5-2.6$. This is to be expected as even though the film is growing at a constant rate without significant compaction, the stress in the film can increase as it grows. The rapid initial rise in ri from 1.5 to 2.1 is consistent with the initial film being more amorphous (typical ri of $a: C 1.8-2.0$ ), ${ }^{8}$ and an increasing diamond (ri of 2.4) or diamond-like content in the film growing on this initial film. For films deposited at -320 and $-160 \mathrm{~V}$ it was found that before an etch/deposition balance was reached the films delaminated due to the stored energy in the thicker films, under high compressive stress. At these dc biases it was possible to continue deposition with constant growth rates for up to $70 \mathrm{~min}$ without delamination taking place.

\section{Substrate surface area}

A substrate surface area dependence on the growth rate, ri and stress in the films has also been observed. Figures 9 and 10 show the variation of thickness and ri with varying substrate area, respectively. The deposition parameters were identical for all substrates, dc bias was maintained at $-450 \mathrm{~V}$, and deposition time was $30 \mathrm{~min}$. The area unit of 1 corresponds to the surface area of a $75-\mathrm{mm}$ diam Si wafer. As can be seen from the data in Fig. 9 the film thickness, and hence growth rate, increases with surface area. Taken together with the ri and stress data in Figs. 10 and 8, it demonstrates that the effective dc bias under which the deposition takes place is influenced by the

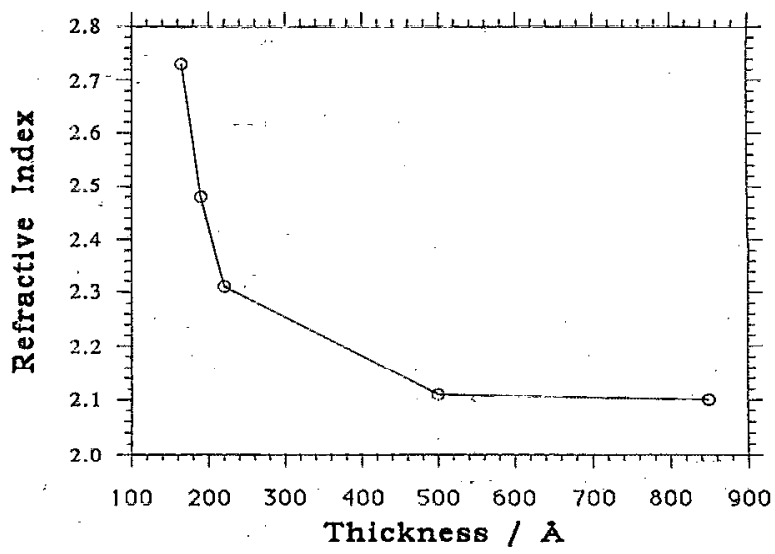

FIG. 10. Refractive index vs thickness.

substrate surface area. Comparing this data with the data in Figs. 5 and 7 where the substrate area is constant and the dc bias varies, it is seen that larger surface area has the same effect as reducing the dc bias. This indicates that the measured dc bias, which is between the plasma and the electrode, is not the sole influence on the electric field which accelerates the ions in the carbon-diamond etch/ deposition process.

The area dependence of this deposition process is illustrated by numerical electric field simulations of the deposition conditions. The influence the substrate area had on the dc electric field was modeled in two dimensions (2-D) by applying $450 \mathrm{~V}$ between the two electrodes held $0.5 \mathrm{~cm}$ apart, this being the approximate height of the sheath space in the if deposition system used. The dielectric constant of the sheath area was taken as 1 . Si substrates (dielectric constant 11.9) of varying length were placed on the "lower electrode." This was held at $-450 \mathrm{~V}$, with the "upper electrode" earthed. Figures 11(a), 11(b), and 11 (c) show contours of constant electric field in $\mathrm{mV} / \mu \mathrm{m}$ for these Si substrates. It can be seen that the electric field across the surface area of the smallest substrate is comparable to those fields that are present at the edges of the larger substrates. This effect is also seen experimentally as a variation in the color of the interference fringes from the edge of the substrate to the middle, for films thicker than $0.4 \mu \mathrm{m}$.

Placing of the Si substrate on the driven electrode gives rise to larger local electric fields as the substrate edges. For small substrates the ions accelerated by this high edge electric field can take part in film growth over the entire surface. However, for large substrates the high edge fields will only influence film growth around the edges and the bulk of the surface will appear an extension of the underlying electrode. Therefore the effective dc bias under which growth takes place will be significantly larger for smaller substrate areas.

From the thickness data of Fig. 9 a critical substrate surface area of $10 \mathrm{~cm}^{2}$ can be obtained. This is the substrate surface area below which edge effects dominate. This has implications for extension of the rf PECVD carbondiamond process for large area coatings of films. A viable 


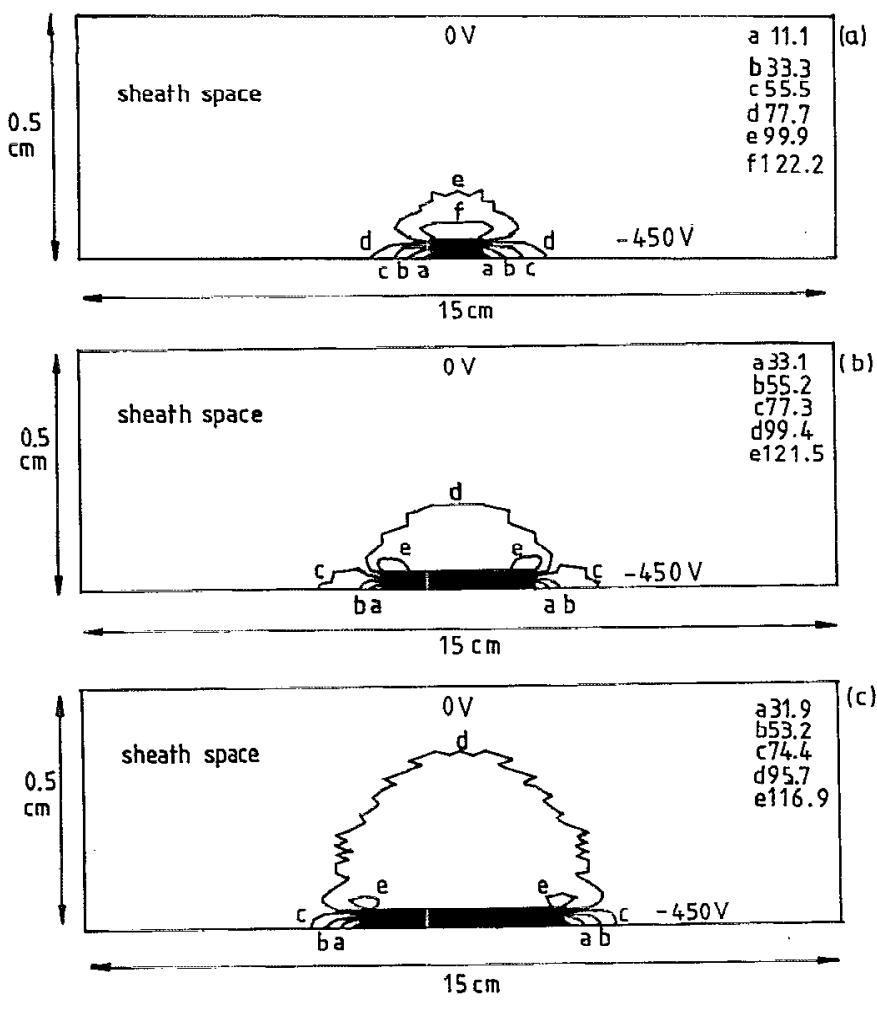

FIG. 11. (a), (b), and (c) Simulated variations of electric field due to $-450 \mathrm{~V}$ dc bias with increasing Si substrate dimensions. Figures show constant electric field contours in $\mathrm{mV} / \mu \mathrm{m}$.

method to overcome this problem would be to control the dc bias independently by placing a grid connected to a separate supply above the substrate.

\section{CONCLUSIONS}

The rf PECVD of carbon-diamond films from a $\mathrm{CH}_{4} / \mathrm{Ar}$ plasma is shown to be a combined etch/ deposition process. The key parameter which influences the etch, and hence deposition rate is the effective dc self bias between the substrate and the plasma. As the film grows a balance in the etch/deposition process can be achieved so that net film growth stops. The film thickness at which such a balance is achieved is also determined by the $\mathrm{dc}$ bias. Once film growth stops the film is subjected to compaction or densification by ion bombardment. This gives rise to an increase in film stress and refractive index. Data which show that the effective dc bias between the substrate and the plasma is a function of substrate surface area is also presented. These results suggest that local edge electric field effects determine the effective dc bias of small substrates.

${ }^{1}$ K. Shenai, R. S. Scott, and B. J. Baliga, IEEE Trans Electron. Dev. 36, 1811 (1989).

${ }^{2}$ F. P. Bowden and D. Tabor, in Physical Properties of Diamond, edited by R. Berman (Clarendon, Oxford, 1965).

${ }^{3}$ C. D. Clark, E. W. J. Mitchell, and B. J. Parsons, in Properties of Diamond, edited by J. E. Field (Academic, New York, 1979).

${ }^{4}$ G. Amaratunga, A. Putnis, K. Clay, and W. Milne, Appl. Phys. Lett. 55,634 (1989).

${ }^{5} \mathrm{M}$. Welland and G. Amaratunga, Surf. Sci. Lett. (to be published).

${ }^{6}$ H. Windischmann and G. F. Epps, J. Appl. Phys. 68, 5665 (1990).

${ }^{7}$ D. R. McKenzie, L. Botten, and R. McPhedran, Phys. Rev. Lett. 51, 280 (1983).

${ }^{8}$ B. Dischler, A. Bubenzer, and P. Koidl, Solid State Commun. 48, 105 (1983).

${ }^{9}$ A. Grill, V. Patel, and B. S. Meyerson, J. Mater. Res. 5, 2531 (1990).

${ }^{10}$ CRC Handbook of Chemistry and Physics, 67th ed., edited by R. C. West (CRC, Boca Raton, FL, 1986).

${ }^{11}$ A. G. Whittaker, Science 200, 763 (1978).

${ }^{12} \mathrm{X}$. H. Wang, L. Pilione, W. Zhu, W. Yarbrough, W. Drawl, and R. Messier, J. Mater. Res. 5, 2345 (1990).

${ }^{13}$ N. Wada, P. J. Gaczi, and S. A. Solin, J. Non-Cryst. Solid. 35/36, 543 (1980).

${ }^{14}$ D. S. Knight and W. B. White, J. Mater. Res. 4, 385 (1989).

${ }^{15}$ D. R. McKenzie, D. Muller, B. A. Pallithorpe, Z. H. Wang, E. Krautchinskia, D. Segal, P. B. Lukins, P. D. Swift, P. J. Martin, G. Amaratunga, A. Saeed, and P. H. Gaskell, Diamond and Related Materials 1, 51 (1991).

${ }^{16}$ D. R. McKenzie, D. A. Muller, E. Krautchinskia, D. Segal, D. H. Cockayne, G. Amaratunga, and R. Silva, Proc. American Vac. Soc., San Diego, April 1991, ICMCTF 1991. 